\title{
Severe hepatitis caused by cyproterone acetate
}

\author{
J C Blake, A M Sawyerr, J S Dooley, P J Scheuer, N McIntyre
}

\begin{abstract}
A case of severe acute hepatitis caused by cyproterone acetate in a $\mathbf{7 1}$ year old man with prostatic carcinoma is reported with a review of the literature on hepatic reactions to this drug. The association between the use of cyproterone acetate and liver abnormalities is poorly documented. This is the fourth published report of adverse hepatic reaction to cyproterone acetate and it substantiates other evidence that cyproterone acetate is potentially hepatotoxic. Monitoring of liver function tests should be mandatory in patients receiving high doses of cyproterone acetate; the drug should be withdrawn immediately if abnormal liver function tests are found.
\end{abstract}

Cyproterone acetate is a widely used potent antiandrogenic drug. It is marketed for use in the treatment of prostatic cancer and advanced breast cancer (Cyprostat; Schering), for hypersexuality and sexual deviation in men (Androcur; Schering), and for hirsutism and severe acne in women (Diane; Schering). Cyproterone acetate is a chlorinated derivative of progesterone and has both antitestosterone and progesterone like activities; it inhibits the peripheral actions of testosterone and suppresses gonadotrophin secretion by maintaining the negative feedback on the pituitary. Liver enzyme abnormalities and hepatic tumours have developed in laboratory animals given cyproterone acetate, ${ }^{1}$ but there have been few reports of liver disease associated with this drug in man.

\section{Case report}

In March 1987 a 71 year old man with a four year history of hesitancy of micturition was treated by transurethral prostatic resection. Adenocarcinoma was found in the prostate tissue removed. Preoperative liver function tests were normal. Soon after the operation he was given cyproterone acetate $300 \mathrm{mg}$ daily. Eight weeks later he complained of nausea, fatigue, and increasing muscle weakness. After 23 weeks of cyproterone acetate treatment he was admitted to hospital with jaundice, swollen legs, and severe muscle cramps. He had been taking propranolol $20 \mathrm{mg}$ daily for 10 years to control supraventricular ectopic beats, and nifedipine $20 \mathrm{mg}$ daily, since a myocardial infarction in 1983 , for the control of hypertension and angina. There was no history of excess alcohol consumption, past administration of blood products or blood transfusion, or previous hepatitis. On examination he was jaundiced and had bilateral leg oedema. The jugular venous pressure was not raised. Abdominal and neurological examinations were normal. Three weeks after admission to hospital he developed a flapping tremor of the hands and became increasingly confused.

The results of investigations were as follows: haemoglobin $12.8 \mathrm{~g} / \mathrm{dl}$; total white cell count $6.6 \times 10^{9} / 1$, with normal differential count; platelet count $155 \times 10^{9} / 1$; blood film showed many target cells; prothrombin time 19 seconds (INR 1.7); ferritin $6600 \mu \mathrm{g} / \mathrm{l}$ (NR 39-340). The results of liver function tests were: bilirubin 183 $\mu \mathrm{mol} / \mathrm{l}$ (NR 5-17), aspartate aminotransferase 672 IU/1 (NR 5-40), alkaline phosphatase 166 IU/l (NR 35-130). Electrolytes, serum albumin, iron, and percentage saturation of total iron binding capacity were normal. Hepatitis B surface antigen, IgM anti-hepatitis B core antibody and IgM anti-hepatitis $A$ antibody tests were negative; there was no serological evidence of recent infection with herpes simplex virus, Epstein-Barr virus or cytomegalovirus. No evidence of tumour in the liver or pelvis was seen on the computed tomography scan, but there was some ascites. An electroencephalogram, performed when the patient was confused, had a mean frequency of 6 cycles per second which is consistent with hepatic encephalopathy.

A liver biopsy performed by the transjugular route because of abnormal haemostasis, showed ballooning of hepatocytes and evidence of regeneration, together with infiltration of the portal tracts by inflammatory cells including neutrophils, eosinophils, and plasma cells. Some cholestasis and bile infarction was also seen. These changes are consistent with a drug idiosyncrasy of predominantly hepatocellular type (Fig 1). In view of the cholestasis in the biopsy an endoscopic retrograde cholangiogram was performed which showed no biliary obstruction.

On admission to hospital all previous drugs were stopped. Initially the patient's condition continued to deteriorate with worsening encephalopathy and the formation of ascites. Serum albumin fell to $26 \mathrm{~g} / \mathrm{l}$. He was treated with bed rest, restriction of dietary sodium, water and protein, and a therapeutic paracentesis of 21 of ascites with intravenous albumin replacement was performed. Spironolactone was required to control fluid retention. Later propranolol and nifedipine were restarted after improvement of liver function tests. $\mathrm{He}$ was not rechallenged with cyproterone acetate. His recovery was prolonged but complete. He was in hospital for six weeks, and liver function tests were normal after nine weeks. (Aspartate aminotransferase and bilirubin concentrations are plotted in Figure 2.)

The case was reported to the Committee on Safety of Medicines as an adverse reaction to cyproterone acetate.

\section{Discussion}

This patient developed severe hepatitis with 


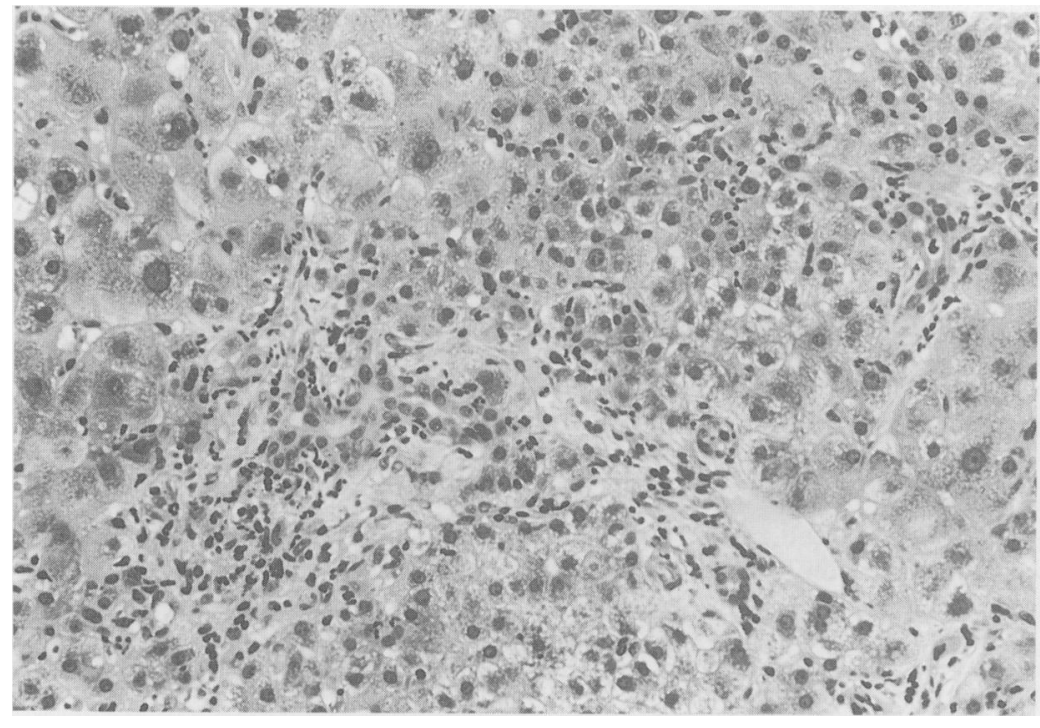

Figure 1: Part of liver biopsy showing hepatitic reaction. The portal tract in the lower half of the figure is expanded, oedematous and inflamed. Many neutrophils are seen around a bile duct as well as within the parenchyma. Liver cells above left and below right are swollen.

Haematoxylin and eosin.

hepatic decompensation after the administration of cyproterone acetate. His recovery after its withdrawal together with the lack of evidence for any other aetiological agent suggests a causal relationship.

There are only three other published reports mentioning adverse hepatic reactions to cyproterone acetate in man. The prospective study of Meijers $e t a l^{2}$ reports abnormal aminotransferase concentrations not attributable to liver metastases or viral hepatitis in five of 20 women treated for advanced breast cancer with cyproterone acetate $200-400 \mathrm{mg}$ daily for six to 52 weeks. Liver histology in the two worst affected patients showed bridging necrosis with evidence of scarring. Levesque $e t a l^{3}$ reported a 78 year old man with carcinoma of the prostate taking cyproterone acetate $300 \mathrm{mg}$ daily who died of fulminant hepatic failure after five months treat-

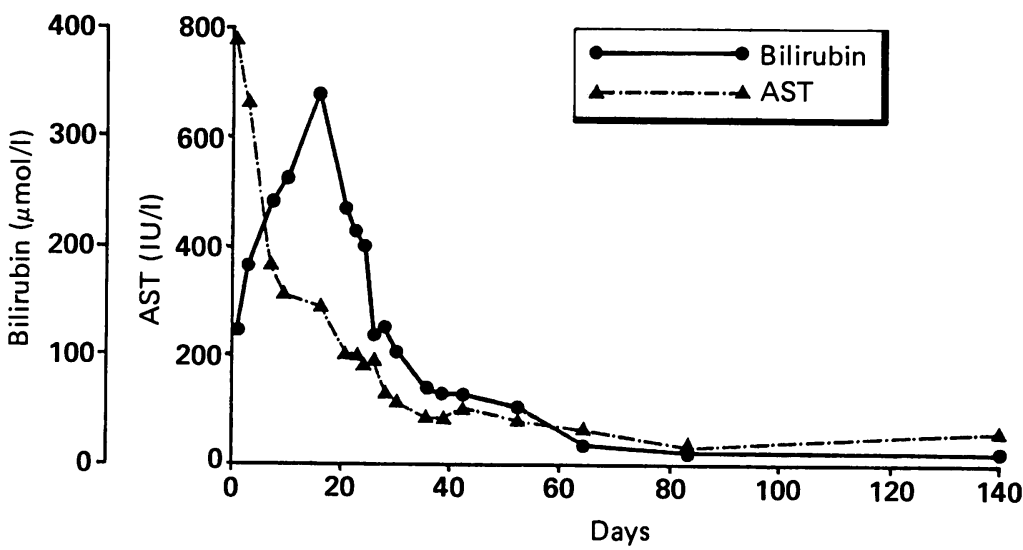

Figure 2: Serum total bilirubin and aspartate aminotransferase (AST) from time of withdrawal of cyproterone acetate (day 0$)$ to normalisation of liver function tests. ment. No other aetiological factors were found in this case. Liver biopsy showed severe necrosis with infiltration by polymorphonuclear cells. Kaiser and Gruner ${ }^{+}$found that four of 77 women taking lower doses of cyproterone acetate (for three to 11 years) for treatment of severe acne, hirsutism or baldness had abnormal liver enzyme concentrations.

Although few case reports have been published, the Committee on Safety of Medicines had received 19 reports in the UK of adverse hepatic reactions associated with cyproterone acetate by November 1988 (personal communication). Five of these cases were fatal. The manufacturers received 153 reports worldwide between 1982 and 1987 of liver abnormalities in patients treated with cyproterone acetate (personal communication), although clinical details of these cases are not known.

The mechanism of toxicity is uncertain. This case has features similar to drug induced acute hepatitis caused by isoniazid and methyldopa: ${ }^{5}$ after treatment for about two to three months, non-specific symptoms develop, including gastrointestinal upset, malaise and muscle weakness. These continue for one to four weeks before the onset of jaundice. The severity of the hepatitis is greatly increased if the drug is continued during the prodrome with a considerable increase in mortality. The toxicity of cyproterone acetate may be direct but is more likely to be the result of an idiosyncratic reaction caused by one of its metabolites. The possibility of an immunological mechanism has not been excluded

Cyproterone acetate is used in a variety of conditions where antiandrogenic effects are beneficial; the recommended dose is between $2 \mathrm{mg}$ and $300 \mathrm{mg}$ daily. The data currently available suggest that adverse hepatic reactions may occur more commonly in elderly patients with malignant disease who are treated with prolonged high doses. Monitoring of liver function tests, which is recommended in the current data sheets of Androcur and Cyprostat, is mandatory in this group of patients and alternative treatment should be found if the results are abnormal.

We wish to thank the Committee on Safety of Medicines and Schering Health Care Limited for providing information on reported reactions to cyproterone acetate.

1 Neumann F. Pharmacology and potential use of cyproterone acetate. Horm Metab Res 1977; 9: 1-13.

2 Meijers WH, Willemse PHB, Sleijfer DTh, Mulder NH, Grond J. Hepatocellular damage by cyproterone acetate. Eur $\mathcal{f}$ Cancer Clin Oncol 1986; 22: 1121-2

3 Levesque H, Trivalle C, Manchon ND, et al. Fulminan hepatitis due to cyproterone acetate. Lancet 1989; i: 215-6.

4 Kaiser E, Gruner HS. Liver structure and function during longterm treatment with cyproterone acetate. Arch Gynecol 1987; 240: $217-23$.

5 Sherlock S. Diseases of the liver and biliary system. Oxford: Blackwell Scientific, 1989: 372-409. 